\title{
Statistical Left Ventricular Deformation Analysis
}

\author{
Si Yong Yeo ${ }^{1}$, Xulei Yang ${ }^{1}$, Like Gobeawan ${ }^{1}$, Soo Kng Teo ${ }^{1}$, Liang Zhong², Ru San Tan², Yi Su${ }^{1}$ \\ ${ }^{1}$ Institute of High Performance Computing, A*STAR, Singapore \\ ${ }^{2}$ National Heart Centre Singapore, Singapore
}

\begin{abstract}
The left ventricular ( $L V$ ) deformation is associated with the LV function, an analysis of which gives insights into the LV mechanics, increasing the diagnostic value of typical parameters like volume, ejection fraction (EF) and strain. This article describes a statistical technique to characterize the LV deformation. 43 cardiac magnetic resonance imaging (MRI) datasets, consisting of normal and diseased $L V$, were used in the experiment. The $L V$ endocardial contours were segmented from the MRI slices across the different frames, and reconstructed as spatiotemporal LV meshes. The geometries were aligned and vertex correspondences between the geometries were computed. The vertex deformation of the LV endocardial surface meshes were then computed at the end-diastolic (ED) and end-systolic (ES) frames. The principal component analysis (PCA) was applied to extract the different modes of variation of the $L V$ deformation. This allows the parametric model to describe the complex $L V$ deformation variation. The experiment shows that there are substantial statistical differences in the $L V$ deformation between the normal and diseased $L V$ geometries. The proposed statistical model can be used to simulate the deformation of the LV geometry, and for the categorization of the LV function in the dataset.
\end{abstract}

\section{Introduction}

The left ventricular (LV) function is typically assessed by parameters such as volume, myocardial thickness, and the qualitative evaluation of the cardiac wall deformation. The analysis of LV deformation can give insight into the mechanics of the normal and diseased left ventricle, and the LV function. Such analysis are based on parameters such as strain, myocardial thickening and rotation, which may not be appropriate to describe the complex myocardial deformation which characterize the LV function.

There are several techniques [1,2] for the analysis of the deformation of LV shape. The finite element model (FEM) has been a widely applied technique [3] for the modelling and analysis of cardiac deformation. Such techniques may however require constitutive modelling which describes the myocardial deformation.

The different parameters of the LV deformation can be used as features for the evaluation of the LV function. Several research techniques have been used in the computational analysis of normal or diseased LV based on the parameters of the $\mathrm{LV}$ deformation. It is however difficult to characterize the differences in the myocardial deformation parameters between the normal and diseased $\mathrm{LV}$ dataset because of the complexity of the LV deformation. In this article, a statistical technique which can characterize the variation of the deformation of the $\mathrm{LV}$ vertices is used for the analysis of the $\mathrm{LV}$ deformation.

The statistical shape models $[5,6]$ have been used to describe the cardiac shape variation. The construction of the shape models are based on the alignment of the cardiac data. The principal component analysis (PCA) technique, a widely used tool for data compression and the extraction of data features, has been used for the modelling of anatomical shapes. Such techniques can be used in the analysis of the shape parameters associated with cardiac disease. The statistical shape model had been incorporated into segmentation techniques $[7,8]$ to constraint the shape variation of the LV geometry. There are however few techniques which are on the statistical analysis of the LV deformation from cardiac MRI dataset. This is because of the complexity in the computation of the vertex correspondence between the LV dataset.

In this article, the PCA technique is used in the analysis of the LV deformation. The LV deformation can therefore be characterized efficiently by a parametric model that describes the LV deformation variation.

\section{Methodology}

In this section, the LV deformation analysis technique is described, which consists of the segmentation of the LV geometry and the computation of vertex correspondences between the different geometries. In particular, the template alignment technique in Su et al. [9] is used in the computation of the vertex correspondences. The statistical analysis of the LV 
deformation.

\subsection{Segmentation of the LV geometries}

The LV geometries for the analysis of the systolic deformation are reconstructed from the LV endocardial boundaries delineated from the cardiac magnetic resonance imaging (MRI) datasets. There are several techniques for the segmentation of LV geometries from biomedical dataset $[10,11]$. It is however difficult for such techniques to segment the LV endocardial boundaries because of the intensity inhomogeneity and noise in the cardiac MRI dataset.

In this article, the $\mathrm{LV}$ endocardial boundaries were manually segmented from contiguous MRI slices across the cardiac cycle. The geometries are then reconstructed from the segmented slices for the different frames. Fig. 1 depicts the segmentation of the LV geometry from the MRI dataset.

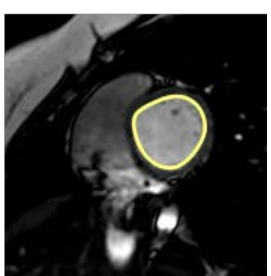

basal

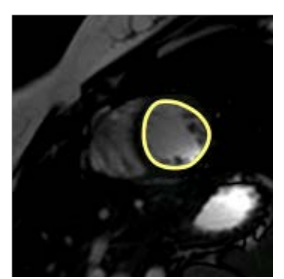

mid

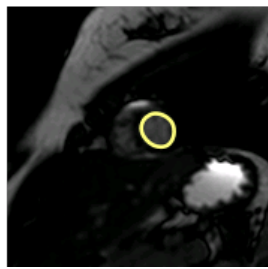

apical (a)

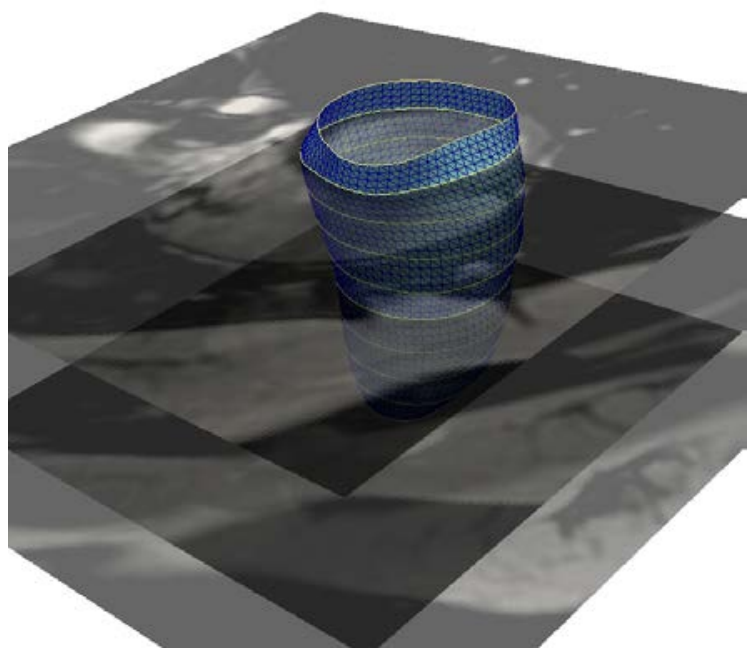

(b)

Figure 1 . The reconstruction of the LV geometry from the MRI dataset, (a) the basal, mid and apical slices, and (b) the extracted LV boundaries and the reconstructed LV geometry.

\subsection{Statistical LV deformation analysis}

In this section, the systolic LV deformation is defined as the difference of the LV geometric vertices between the end-diastolic and systolic, i.e. ED and ES frames. Therefore, vertex correspondences of the LV geometries between different frames have to be computed in order to perform the LV deformation analysis. To do so, a template alignment technique is used, in which a template mesh with fixed number of vertices and of fixed topology is aligned to the reconstructed geometry of the LV endocardial surface. The radial-basis-function based coarse matching process is used to transform the template mesh to an approximate shape of the LV endocardial surface. Next, a fine matching process based on an optimization technique adapts the position of the template mesh vertices so that they conform closely to the actual geometry. As the optimization function is based on a strain energy function, the outcome exhibits superior mesh quality with plausible LV deformation. Since the same template mesh is used in the alignment of the different frames, the vertex correspondence between the $\mathrm{LV}$ frames is established across the LV dataset, hence facilitating statistical analysis.

There can be considerable geometric deformation of the LV from ED to ES. In particular, the systolic LV deformation can consist of radial and torsional components. As such, the complex LV geometries are described by the cylindrical coordinate system defined locally at the mean vertex positions of the different $\mathrm{LV}$ regions, i.e., the basal, mid and apical regions. Fig. 2 depicts the systolic deformation of a normal LV geometry. It is shown that there is a significant difference between the geometries of the LV at the ED and ES frames.

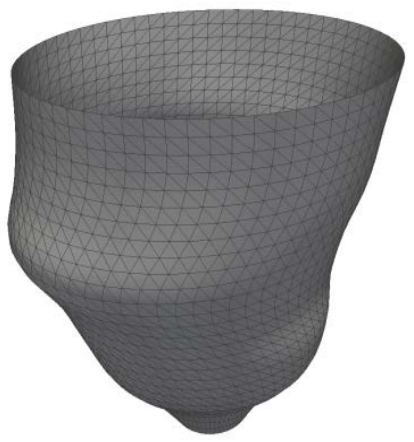

ED

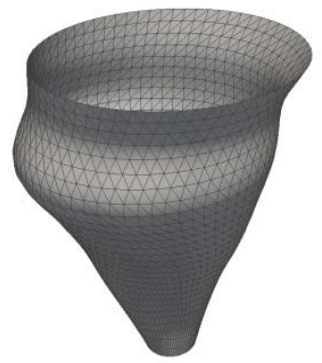

ES
Figure 2. The LV geometries at the ED and ES frames.

The geometries of the LV regions at the ED and ES frames of the different samples are aligned to minimize the differences caused by the rigid transformation, i.e., translation and rotation. 
This allows the geometric vertices of the $\mathrm{LV}$ to be defined as $\mathrm{v}=(\mathrm{r}, \theta, \mathrm{z})$. The $\mathrm{LV}$ deformation can therefore be described by the radial, circumferential and longitudinal components. The deformation vector of the vertices between the frames can then be computed as

$$
\mathbf{x}=\mathbf{v}_{E D}-\mathbf{v}_{E S}
$$

The PCA technique can therefore be applied to compute the $L V$ deformation variation.

Consider a set of $\mathrm{n}$ shapes, and each shape consists of $\mathrm{m}$ vertices. The deformation vectors computed at the vertices of each of the shape can therefore be denoted by $\left\{\mathrm{x}_{\mathrm{i}} ; \mathrm{i}=1 \ldots m\right\}$. The vectors can be approximated by a linear model,

$$
\mathbf{x}=\overline{\mathbf{x}}+\Phi \mathbf{b}
$$

in which $\overline{\mathbf{x}}=\frac{1}{n} \sum_{i=1}^{n} \mathbf{x}_{i}$ is the mean deformation vector, $\mathbf{b}$ is the weight vector of the model, and $\Phi$ is composed of the eigenvectors, computed by performing a PCA on the covariance matrix

$$
\boldsymbol{S}=\frac{1}{n-1} \sum_{i=1}^{n}\left(\mathbf{x}_{i}-\overline{\mathbf{x}}\right)\left(\mathbf{x}_{i}-\overline{\mathbf{x}}\right)^{T}
$$

The vector $\mathbf{b}$ denotes the deformation parameters, and can be varied to approximate the different LV deformation in the training set. The deformation vector can therefore be used to synthesize the systolic deformation of a LV shape.

The complex LV deformation can therefore be described as a linear combination of the modes of deformation. This allows the LV deformation to be characterized by a parametric model that describes the variation of the deformation of the LV vertices of the different dataset. The deformation parameters can be used in the categorization of the normal and diseased LV. In particular, a linear support vector machine (SVM) is trained using the PCA transformed data for the categorization of the normal and diseased LV dataset. The $\mathrm{k}$-fold cross-validation is used to compute the accuracy of the SVM model in the categorization of the LV dataset.

\section{Results and Discussion}

This section describes the experiment and analysis of the LV deformation based on the statistical technique. In particular, 43 cardiac MRI datasets, consisting of 8 normal and 35 diseased LV, were used in the experiment.

Significant statistical differences were observed between the normal and diseased LV deformations. It is shown that $87 \%$ of the variation in the LV deformation can be described by the five most significant modes of variation, i.e. the modes of variation which show the largest variance in the data. Figure 3 depicts the plot of the weight vector of the three most significant modes of deformation for the 43 datasets.

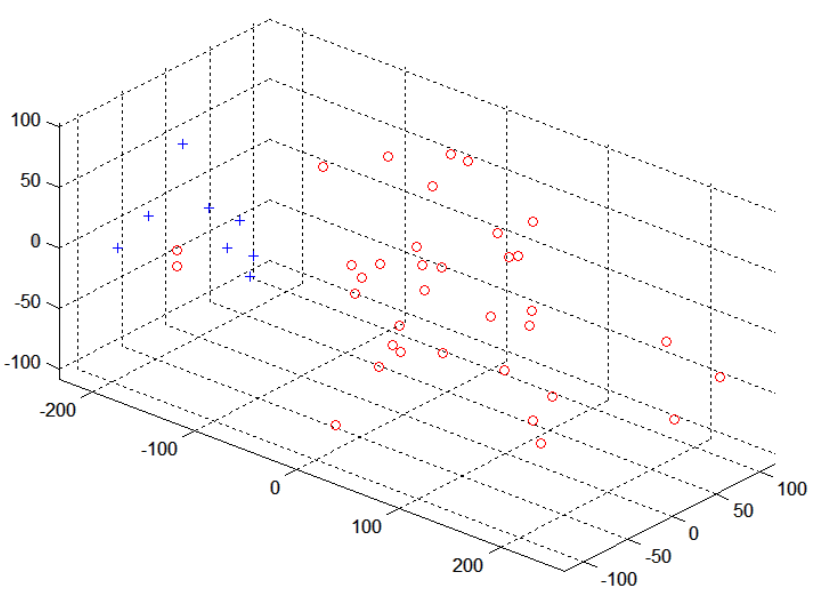

Figure 3. The plot of the weight vectors of the three most significant modes of deformation of the 43 datasets, depicting the weight vectors of the normal (blue) and diseased (red) LV deformation.

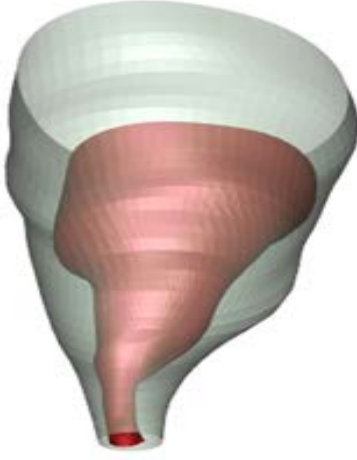

$-3 \sigma$

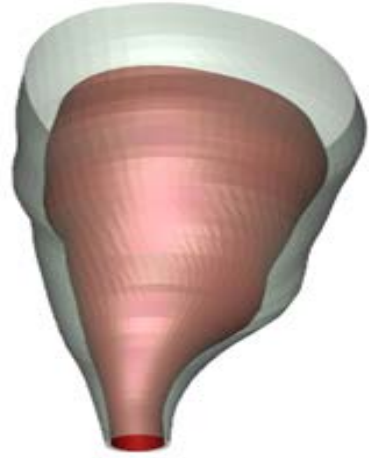

mean

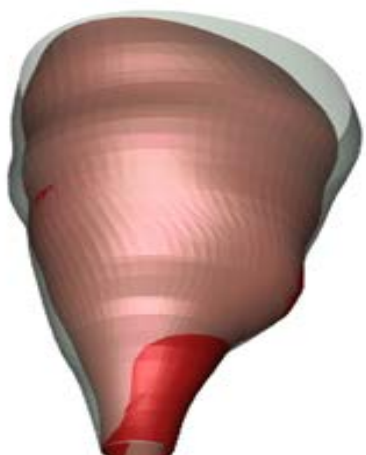

$3 \sigma$

Figure 4. The LV geometry at the ED frame (grey), and the simulated LV geometries at the ES frame (red) by a single deformation component, in which $\sigma$ denotes the standard deviation. The mean LV deformation and deformation vectors which are $3 \sigma$ from the mean are applied on the LV geometry at the ED frame. 
It is shown that the weight vectors for the normal and diseased LV tend to cluster differently.

The statistical model can therefore efficiently characterize the variation of the systolic deformation of the LV geometries. Fig. 4 depicts a sample that exhibits the most significant mode of variation of LV deformation. As shown in the figure, the mean LV deformation, and deformation vectors which are $3 \sigma$ from the mean are applied on the LV geometry at the ED frame to simulate the LV geometry at the ES frame. There is a significant difference between the simulated LV geometries at the ES frame. This is caused by the differences in the normal and diseased LV deformations.

The PCA transformed data were used as features for training a linear SVM to categorize the normal and diseased LV. The SVM analysis on the LV datasets yielded a classification accuracy of 92.5\%. The SVM model could accurately distinguish the normal LV from the diseased LV with normal EF. This shows that a robust method can be derived from the statistical model of LV deformation to assess the LV function. Such a technique can be used in the analysis of the LV deformation variation in the different cardiac dataset.

\section{Conclusions}

This article described a LV systolic function analysis technique based on statistical representation of LV deformation. The technique consists of the segmentation of the LV geometry, the computation of vertex correspondences between the different geometries, and the statistical analysis of the LV deformation. The PCA technique was applied in the analysis of the modes of variation of the LV deformation. The complex LV deformation can therefore be described as a linear combination of these modes of deformation. This allows the construction of a parametric model to describe the complex LV deformation variation. The statistical model can be used to simulate the deformation of the LV geometry. This shows that the statistical model can efficiently characterized the systolic deformation of a wide variation of the $\mathrm{LV}$ dataset.

\section{References}

[1] Buss S. J., Schulz F., Mereles D., Hosch W., Galuschky C., Schummers G., Stapf D., Hofmann N., Giannitsis E., Hardt S. E., Kauczor H. U., Katus H. A., Korosoglou G., Quantitative analysis of left ventricular strain using cardiac computed tomography, Eur J Radiol., 2014, 83(3), 123-30.

[2] Park, J., Metaxas, D. N., Young, A. A., Deformable models with parameter functions: Application to heart-wall modeling, Proceedings of the IEEE Conference on Computer Vision and Pattern Recognition, 1994, 437-442.

[3] Needleman A., Rabinowitz S. A., Bogen D. K., McMahon T. A., A finite element model of the infarcted left ventricle, J. Biomech., 1983, 16(1), 45-58.
[4] Hoogendoorn C., Duchateau N., Sánchez-Quintana D., Whitmarsh T., Sukno F.M., De Craene M., Lekadir K., Frangi A.F.. A high-resolution atlas and statistical model of the human heart from multislice CT. IEEE Trans Med Imaging. 2013, 32(1):28-44.

[5] C.G. Fonseca, M. Backhaus, D.A. Bluemke, R.D. Britten, J.D. Chung, B.R. Cowan, I.D. Dinov, J.P. Finn, P.J. Hunter, A.H. Kadish, D.C. Lee, J.A.C. Lima, P. Medrano-Gracia, K. Shivkumar, A. Suinesiaputra, W. Tao, and A.A. Young. The Cardiac Atlas Project - An imaging database for computational modeling and statistical atlases of the heart. Bioinformatics, 2011,27(16), 2288-2295.

[6] S. Ordas, E. Oubel, R. Leta, F. Carreras. A.F. Frangi. A statistical shape model of the heart and its application to model-based segmentation, Proceedings of SPIE Medical Imaging, 2007.

[7] S. Zhang, M. Uzunbas, Z. Yan, M. Gao, J. Huang, D. N. Metaxas, L. Axel. Construction of Left Ventricle 3D Shape Atlas from Cardiac MRI., Functional Imaging and Modeling of the Heart 2011:88-94.

[8] Frangi A.F., Rueckert D., Schnabel J.A., Niessen W.J.. Automatic construction of multiple-object threedimensional statistical shape models: application to cardiac modelling. IEEE Trans Med Imaging 2002, 21(9),11511166.

[9] Su Y., Teo S.-K., Lim C. W., Zhong L., Tan R. S., Automatic generation of endocardial surface meshes with 1-to-1 correspondence from cine-MR Images, Proc. SPIE Medical Imaging, Computer-Aided Diagnosis, Orlando, Florida, USA, 2015, 21-26.

[10] Santarelli M. F., Positano V., Michelassi C., Lombardi M., Landini L., Automated cardiac MR image segmentation: Theory and measurement evaluation, Med. Eng. Phys., 25(2), 149-159, 2003.

[11] Cremers D., Kohlberger T., Schnoerr C., Shape statistics in kernel space for variational image segmentation, Pattern Recognition, 2003, 36, 1292-1943.

Address for correspondence.

Yi Su

Institute of High Performance Computing, A*STAR

1 Fusionopolis Way, Connexis (North) \#16-16

Singapore 138632

suyi@ihpc.a-star.edu.sg 